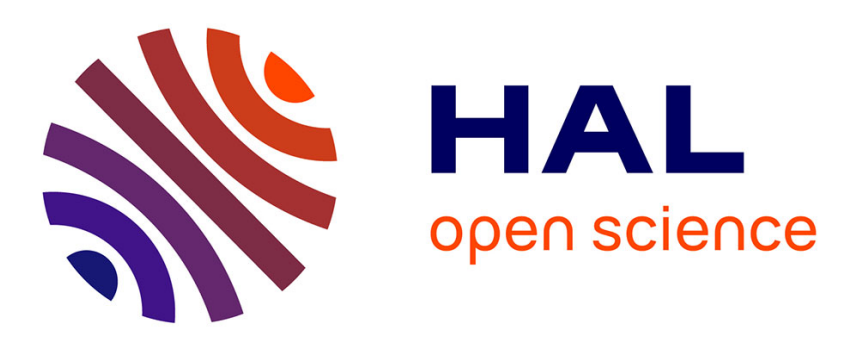

\title{
Les nouveaux colzas à basse teneur en glucosinolates: composition chimique et valeur nutritive des tourteaux et graines pour le porc \\ D. Bourdon
}

\section{- To cite this version:}

D. Bourdon. Les nouveaux colzas à basse teneur en glucosinolates: composition chimique et valeur nutritive des tourteaux et graines pour le porc. Productions Animales, 1989, 2 (4), pp.267-273. hal00895874

\section{HAL Id: hal-00895874 \\ https://hal.science/hal-00895874}

Submitted on 1 Jan 1989

HAL is a multi-disciplinary open access archive for the deposit and dissemination of scientific research documents, whether they are published or not. The documents may come from teaching and research institutions in France or abroad, or from public or private research centers.
L'archive ouverte pluridisciplinaire HAL, est destinée au dépôt et à la diffusion de documents scientifiques de niveau recherche, publiés ou non, émanant des établissements d'enseignement et de recherche français ou étrangers, des laboratoires publics ou privés. 
INRA Prod. Anim., 1989, 2 (4), 267-273

\section{BOURDON}

INRA Station de Recherches porcines Saint-Gilles 35590 L'Hermitage

\section{Les nouveaux colzas à basse teneur en glucosinolates :}

\author{
composition chimique \\ et valeur nutritive des tourteaux \\ et graines pour le porc
}

La teneur élevée en glucosinolates constitue un facteur limitant majeur à l'utilisation des graines et tourteaux de colza dans l'alimentation du porc. En effet leurs produits de dégradation, générés au cours du traitement technologique classiquement pratiqué en huilerie, sont responsables d'effets néfastes : inappétence et désordres physiologiques divers... La création de nouvelles variétés de colza à basse teneur en glucosinolates, encore appelées double zéro, associée à l'amélioration des traitements technologiques appliqués aux graines permet d'accroître la valeur nutritive et les taux d'incorporation du tourteau de colza (jusqu'à 10-20\%) dans les aliments pour le porc à l'engrais.

La production française de graines de colza a connu au cours des dix dernières années un développement spectaculaire : de 568000 tonnes en 1978, elle a atteint 2500000 tonnes produites sur 868500 ha en 1988. Le colza constitue ainsi la première source française pour la fourniture d'huile et de tourteau.

\section{Résumé}

Les données de valeur nutritive (valeurs énergétique et azotée) de 30 échantillons de tourteaux et graines de colza à haute (HG) et basse (BG) teneur en glucosinolates, obtenues par mesure de digestibilité directe sur porc à l'engrais, sont présentées par type de tourteau (extrait au solvant, de pression, normal et dépelliculé), de même que pour les graines traitées. Nos résultats expérimentaux sont comparés à un nombre équivalent de données bibliographiques disponibles. Ils permettent de mieux préciser, pour le porc, la valeur nutritive des produits dérivés du colza et notamment des nouveaux tourteaux (BG) sous forme normale et dépelliculée.

Les teneurs en glucosinolates des graines de colza et tourteaux dérivés (HG) et (BG) ayant été précisées, divers traitements technologiques sont évalués en vue d'améliorer la valeur nutritionnelle des tourteaux et graines de colza (BG).

Ces données mettent en évidence, la marge de progrès possible avec l'utilisation de tourteau (BG) à basse teneur en glucosinolates, dépelliculé dont la valeur nutritive et les possibilités d'utilisation sont nettement accrues pour le porc à l'engrais.

Les réserves souvent émises vis-à-vis de l'utilisation des produits du colza se justifient de moins en moins, compte tenu des améliorations acquises ou envisageables à court terme dans les domaines de l'amélioration génétique, des traitements technologiques et de la connaissance de la valeur nutritionnelle des tourteaux.

Ainsi la sélection pour l'amélioration de la qualité de l'huile a créé rapidement, en moins de dix ans, des colzas à faible teneur en acide érucique, mais à teneur élevée en glucosinolates, qualifiés de simple 0 (HG). Par contre l'amélioration de la qualité du tourteau, initiée depuis une dizaine d'années, s'avère plus délicate. Elle nécessite la création de nouvelles variétés à faible teneur en acide érucique et en glucosinolates qualifiées de double 0 (BG).

Cet objectif a été facilement atteint au Canada avec le colza de printemps de type Cinolid. Cette reconversion constitue un objectif prioritaire de la recherche sur le colza, en cours de réalisation à l'heure actuelle en Europe avec les variétés d'hiver. Au plan français des progrès significatifs ont déjà été obtenus par la Station d'Amélioration des Plantes du Centre INRA de Rennes, en collaboration avec les établissements Ringot : deux variétés de colza BGTANDFM et DARMOR - ont été inscrites dès 1983. Compte tenu des progrès possibles, la mise sur 
le marché de variétés de colza BG renfermant moins de 20 micromoles de glucosinolates/g de graine entière est envisagée dès 1989.

Le porc est un consommateur potentiel privilégié des nouveaux tourteaux BG. Nous avons donc étudié la valeur nutritive des nouveaux tourteaux et graines BG, en référence aux mêmes produits issus de colza classique $\mathrm{HG}$. Les différentes voies d'amélioration de la valeur nutritive des tourteaux BG ont également été envisagées.

Après un rappel des teneurs en glucosinolates des produits dérivés et des principales variétés de colza, nous présenterons les caractéristiques de composition chimique des produits testés au cours de nos expériences puis les résultats relatifs à leur valeur nutritionnelle chez le porc.

Ces travaux ont été réalisés en collaboration étroite avec nos collègues de l'amélioration des plantes oléagineuses de l'INRA de Rennes, du CETIOM (Centre Technique Interprofessionnel des Oléagineux Métropolitains) et de l'ONIDOL (Organisation Nationale Interprofessionnelle des Oléagineux) pour les aspects technologiques et de l'Industrie de l'alimentation animale.

\section{1 / Teneurs en glucosinolates et en produits dérivés}

Comme chez toutes les crucifères, la fraction glucidique du colza renferme des composés soufrés : les glucosinolates. Sous l'action d'une enzyme, la myrosinase présente dans la graine et libérée lors du broyage, les glucosinolates s'hydrolysent en isothiocyanates (ITC), vinylthiooxazolidone (VTO), nitriles, thiocyanates... Ces divers produits sont présents en quantité variable selon les variétés. Ils engendrent des effets néfastes (inappétence, toxicité, et actions physiologiques particulières), auxquels le porc se révèle particulièrement sensible (Calet 1982 , Fenwick 1982, Lee et Hill 1983, Bell 1984, Lee et al 1984).

\section{1 / Graines non traitées}

Au tableau 1, figurent les teneurs en glucosinolates totaux des graines de colza de différentes variétés. Les teneurs en glucosinolates totaux varient essentiellement selon

- le génotype à haute (HG) ou basse (BG) teneur en glucosinolates,

- le type de variété d'hiver ou de printemps,

- l'espèce Napus ou Campestris.

Les colzas européens d'hiver, tous d'espèce Napus, présentent des teneurs en glucosinolates plus élevées. Cependant les teneurs des futures variétés françaises de colza d'hiver $\mathrm{BG}$, disponibles pour la campagne 89-90, seront inférieures à 40 micromoles/g de matière sèche délipidée soit moins de 20 micromoles pour la graine entière, en conformité avec la norme CEE exigible dès 1991.

Pour les variétés canadiennes exclusivement de printemps, les espèces Campestris renferment en moyenne moins de glucosinolates que les espèces Napus. Les nouvelles variétés de colza canadiennes CANor $A$ BG des espèces Campestris et Napus présentent des teneurs en glucosinolates totaux réduites 3 à 6 fois comparativement aux variétés HG.

Les graines de quelques variétés européennes de printemps, comme Erglu et Llink d'origines allemande et danoise, présentent de très faibles teneurs en glucosinolates ( 8 à 15 micromoles). Cependant, la recherche d'une très basse teneur en glucosinolates peut entraîner certaines déficiences au plan agronomique, notamment un rendement très faible, comme cela est observé pour le géniteur commun à toutes les variétés de colza BG. Bronowski.

\section{2 / Tourteaux}

Dans le cas des tourteaux, le nutritionniste ne s'intéresse pas seulement à la teneur en glucosinolates totaux, mais également aux quantités de composés formés au cours du processus technologique qui en conditionnent l'appé-

Tableau 1. Teneurs en glucosinolates de colzas à haute (HG) et basse teneur en glucosinolates (en micromoles/g MS délipidée). (D'après Chone 1983, Menzel 1983, Bell 1984, Bourdon 1986)

\begin{tabular}{|c|c|cc|cc|}
\hline $\begin{array}{c}\text { Origine } \\
\text { botanique }\end{array}$ & $\begin{array}{c}\text { Types de } \\
\text { variétés }\end{array}$ & Nom & $\begin{array}{c}\text { HG gluco- } \\
\text { sinolates }\end{array}$ & Nom & $\begin{array}{c}\text { BG gluco- } \\
\text { sinolates }\end{array}$ \\
\hline $\begin{array}{c}\text { B. Campestris } \\
\text { ou } \\
\text { B. Napus }\end{array}$ & $\begin{array}{c}\text { Canadiennes } \\
\text { de } \\
\text { Printemps }\end{array}$ & Torch & 93 & Canola & 28 \\
& $\begin{array}{c}\text { Canadiennes } \\
\text { de } \\
\text { Printemps }\end{array}$ & Midas & $154^{*}$ & Regent & $26^{*}$ \\
\cline { 2 - 6 } B. Napus & $\begin{array}{c}\text { Européennes } \\
\text { de }\end{array}$ & & & Erglu & $15^{*}$ \\
& Printemps & Line & $8^{*}$ \\
\cline { 2 - 5 } & Européennes \\
d'Hiver & Diamant & 156 & Tandem & $59^{*}$ \\
& Jet Neuf & $169^{*}$ & Darmor & $41^{*}$ \\
\hline
\end{tabular}

* Ces valeurs n’incluent par los indolmélhylglucosinolites. 
Tableau 2. Teneurs en glucosinolates et produits dérivés des graines et tourteaux de colza (moyennes et valeurs extrêmes $/ g$ MS délipidée). ITC = isothiocyanates, VTO = vinylthiooxazolidone.

\begin{tabular}{|c|c|c|c|}
\hline Type de produit & ITC mg & VTO mg & $\begin{array}{c}\text { Glucosinolates } \\
\text { totaux } \\
\text { micromoles }\end{array}$ \\
\hline $\begin{array}{l}\text { Tourteaux d'extraction } \\
\text { - HG }(\mathrm{n}=7) \\
-\mathrm{BG}(\mathrm{n}=7)\end{array}$ & $\begin{array}{c}3,5 \\
(2,1-5,5) \\
1,3 \\
(0,3-2,1) \\
\end{array}$ & $\begin{array}{c}9,2 \\
(5,2-14,9) \\
2,7 \\
(0,8-4,6) \\
\end{array}$ & $\begin{array}{c}166^{*} \\
(125-207) \\
38^{\star} \\
(9-69) \\
\end{array}$ \\
\hline $\begin{array}{l}\text { Graines entières } \\
\text { traitées ** } \\
\quad-B G(n=2)\end{array}$ & 1,4 & $\begin{array}{c}2,8 \\
(2,7-2,8)\end{array}$ & $\begin{array}{c}36^{*} \\
(35-37) \\
\end{array}$ \\
\hline $\begin{array}{l}\text { Tourteaux de pression } \\
\text { expellers } \\
-B G(n=5)\end{array}$ & $\begin{array}{c}1,3 \\
(1,3-1,4) \\
\end{array}$ & $\begin{array}{c}3,5 \\
(3,2-3,8)\end{array}$ & $\begin{array}{c}36^{*} \\
(34-38)\end{array}$ \\
\hline $\begin{array}{l}\text { Tourteaux dépelliculés } \\
\text { d'extraction } \\
\text { - BG }(n=5)\end{array}$ & $\begin{array}{c}\mathbf{0 , 8} \\
(0,2-1,5)\end{array}$ & $\begin{array}{c}1,6 \\
(0,3-3,7)\end{array}$ & $\begin{array}{c}30^{\star} \\
(3-49)\end{array}$ \\
\hline
\end{tabular}

( $\mathrm{n}$ - nombre d'échantillons testés)

* Ces valeurs n'incluent par les indolméthylglucosinolates.

** Traitements appliqués = broyage + chauffage modéré aux microondes.

tence et/ou la toxicité. Parmi les tourteaux extraits au solvant, qui représentent le produit le plus courant, ceux issus de colza BG, essentiellement de variété TANDHM présentent des teneurs en ITC, VTO et glucosinolates totaux respectivement 2,$7 ; 3,4$ et 4,4 fois plus faibles que ceux issus de colza HG Jwr Neur (tableau 2).

Certains tourteaux HG expérimentés au cours de nos études furent mal consommés, voire refusés, lorsqu'ils étaient incorporés à taux élevé dans le régime $(20 \%)$. En revanche, tous les tourteaux BG furent bien consommés même incorporés à $20 \%$ dans le régime. Il est bon de préciser que ces observations étaient réalisées au cours d'essais de digestibilité, avec des régimes distribués sous forme humide ce qui place les porcs dans des conditions d'alimentation très discriminantes pour juger de l'appétence de ce type de produit.

Trois produits moins courants dérivés de colza BG ont été également étudiés : il s'agit de graines entières traitées, de tourteaux de pression expellers et de tourteaux dépelliculés extraits au solvant (tableau 2). Leurs teneurs en ITC, VTO et glucosinolates sont comparables à celles des tourteaux BG précédents. Au cours des expériences réalisées sur le porc à l'engrais, ces trois produits, incorporés à taux élevé $\{20 \%\}$ dans le régime furent très bien consommés.

\section{2 / Valeur nutritive chez le porc}

Chez le porc à l'engrais, le second facteur limitant à l'emploi du tourteau de colza est sa faible valeur nutritive comparativement aux sources de protéines usuelles, tourteau de soja, pois, etc. La teneur élevée en cellulose brute (11 à $15 \%$ ) et la richesse en tanins réduisent la digestibilité des fractions énergétiques et azo- tées. En outre, le tourteau de colza présente une teneur en lysine plus faible que le tourteau de soja. Cette déficience relative en lysine est bien souvent accentuée du fait d'une disponibilité réduite de cet acide aminé, suite à l'application d'un traitement thermique trop prononcé en vue d'éliminer ou de neutraliser le maximum de composés dérivés des glucosinolates (Baudet et al 1987).

Nous présentons par catégorie de produits, les résultats moyens de valeur énergétique et azotée, obtenus par mesure directe sur porc à l'engrais en comparaison avec les données bibliographiques disponibles.

\section{1 / Tourteaux de colza extraits au solvant}

(tableau 3)

\section{a / Tourteaux simple 0-HG}

Les huit tourteaux HG que nous avons testés sur porc en croissance et/ou en finition présentent une valeur moyenne du CUDa de l'énergie quasiment similaire à celle de la bibliographie $[68,5 \%$ et $68 \%)$ pour une teneur moyenne en énergie digestible inférieure de $4 \%$ (3 206 et $3343 \mathrm{kcal} / \mathrm{kg} \mathrm{MS}$ ) et en énergie métabolisable voisine (2974 et $2995 \mathrm{kcal} / \mathrm{kg} \mathrm{MS}$ ). Les traitements technologiques, le taux d'introduction du tourteau dans les régimes expérimentaux et l'âge des porcs peuvent être à l'origine de telles différences mineures.

\section{b / Tourteaux double 0-BG}

Les données moyennes de valeur nutritive issues de la bibliographie relatives à 15 tourteaux expérimentaux, diffèrent assez peu de celles rapportées précédemment pour les tourteaux de colza HG. On observe cependant une légère amélioration de la digestibilité de l'éner-
Les produits de dégradation des glucosinolates, ITC et VTO, conditionnent l'appétence et la toxicité des graines et tourteaux de colza. Les variétés double zéro en contiennent peu et sont bien consommées par le porc à I'engrais. 
Tableau 3. Composition chimique et valeur nutritive des tourteaux de colzas simple 0-HG et double $0-B G$ extraits au solvant (moyennes \pm Ecarts-type de la moyenne).

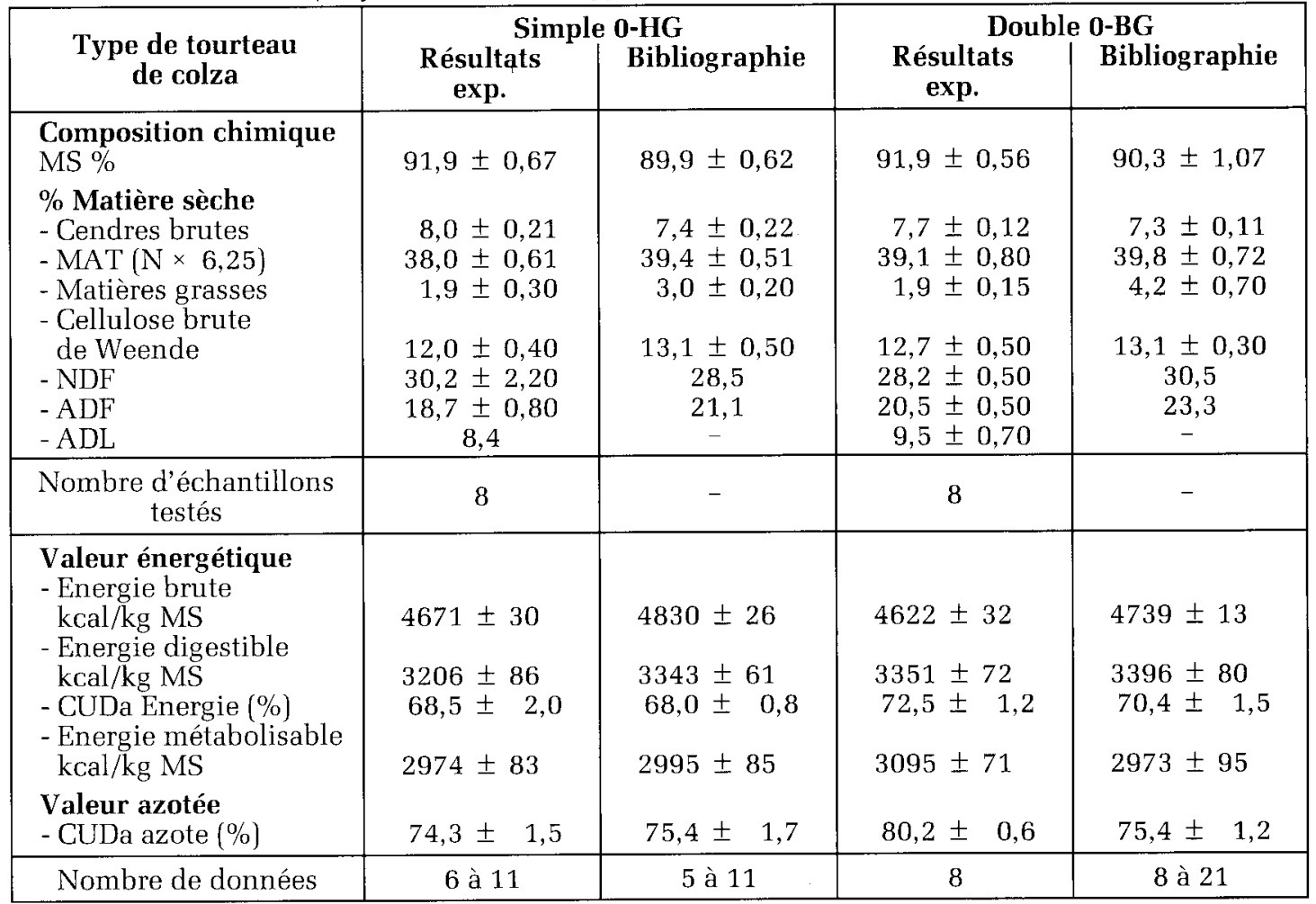

NDF : Neutral Detergent Fiber, ADF : Acid Detergent Fiber, ADL : Acid Detergent Lignin, CUDa : Coefficient d'Utilisation Digestive apparent.

gie $(70,4$ contre $68,0 \%)$ sans toutefois que le contenu en énergie digestible diffère, du fait d'une teneur moyenne en énergie brute réduite de $2 \%$ (4 739 contre $4830 \mathrm{kcal} / \mathrm{kg}$ MS). Mais de nombreux résultats sont obtenus avec des tourteaux issus de graines de variété Bronowsкi, géniteur originel des nouvelles variétés de colza BG qui diffère notablement de ces dernières.

Nos résultats expérimentaux, obtenus sur 8 échantillons de tourteaux issus de graines de nouvelles variétés de colza BG (REGENT et TAN$D E M)$, reflètent beaucoup mieux ce que l'on est en mesure d'obtenir avec les nouveaux tourteaux de colza. En accord avec les résultats de Rundgren 1983, les tourteaux de colza BG présentent en moyenne des caractéristiques nutritives légèrement plus favorables que leurs homologues $\mathrm{HG}$ : $+5,8 \%$ pour le CUD de l'énergie, $+4,5 \%$ pour l'énergie digestible, $4,1 \%$ pour la teneur en énergie métabolisable et $7,9 \%$ pour le CUDa de l'azote. Toutefois ces légères différences peuvent résulter de la meilleure précision de la mesure de la valeur nutritive des tourteaux BG, du fait d'un taux d'incorporation plus élevé dans les régimes expérimentaux $(20 \%$ au lieu de $10 \%$ ). La technologie mise en œuvre peut également expliquer en partie les améliorations observées : une cuisson moins poussée entraîne un accroissement sensible de la digestibilité de l'énergie et de l'azote du tourteau (Baudet et al 1987). Selon les données rapportées par Picard et Darcy-Vrillon (1985), les tourteaux HG et BG présentent des compositions en acides aminés similaires.
Ainsi, lorsqu'ils sont élaborés selon la même technologie, les tourteaux BG et HG diffèrent peu en ce qui concerne leurs caractéristiques nutritionnelles. Même s'ils sont nettement mieux consommés et tolérés par les porcs, la valeur énergétique ainsi que les teneurs en protéines et en lysine des tourteaux BG restent faibles comparativement au tourteau de soja.

\section{2 / Graines de colza entières}

L'utilisation de graines de colza entières, traitées selon divers procédés technologiques est possible chez le porc et le porcelet. Au tableau 4 figurent les données moyennes de composition chimique et de valeur nutritive de graines de colza traitées. Compte tenu de leurs caractéristiques nutritives, Ies graines de colza sont essentiellement destinées au porcelet, exigeant un régime riche en énergie, et à un moindre degré au porc en croissance. Pour ce dernier, le taux d'incorporation doit être plus modéré afin d'éviter de dégrader le degré de saturation du gras. Nos données expérimentales concordent avec les données moyennes de la bibliographie, à l'exception d'une teneur en énergie brute un peu plus faible (6 $502 \mathrm{kcal} / \mathrm{kg}$ MS contre 7066 $\mathrm{kcal} / \mathrm{kg}$ MS) vraisemblablement en raison d'une perte d'huile en cours de broyage. Après un aplatissage et un chauffage modéré, les graines de colza BG présentent, pour le porc à l'engrais, une teneur moyenne en énergie digestible qui s'élève à $5400 \mathrm{kcal} / \mathrm{kg} \mathrm{MS}$, associée à une digestibilité moyenne de la fraction azotée de $83,7 \%$. 


\begin{tabular}{|c|c|c|}
\hline Type de colza & $\begin{array}{l}\text { B. Campestris } B \text {. Napus } \\
\text { Simple 0-HG } \\
\text { Double 0-BG }\end{array}$ & $\begin{array}{c}\text { B. Napus } \\
\text { Double 0-BG } \\
\text { Tandem }\end{array}$ \\
\hline Traitement technologique & $\begin{array}{l}\text { Divers, extrusion, } \\
\text { micronisation, etc }\end{array}$ & $\begin{array}{c}\text { Aplatissage + chauffage } \\
\text { modéré }\end{array}$ \\
\hline $\begin{array}{l}\text { Composition chimique } \\
\text { MS (\%) } \\
\% \text { Matière Sèche } \\
\text { - Cendres brutes } \\
\text { - MAT }(N \times 6,25) \\
\text { - Matières grasses } \\
\text { - Cellulose brute de Weende } \\
\text { - NDF } \\
\text { - ADF } \\
\text { - ADL }\end{array}$ & $\begin{aligned} 93,5 & \pm 0,85 \\
4,3 & \pm 0,34 \\
22,7 & \pm 0,56 \\
42,1 & \pm 1,24 \\
10,1 & \pm 0,83 \\
& - \\
& - \\
- & \end{aligned}$ & $\begin{array}{r}96,8 \\
\\
4,5 \\
23,0 \\
44,4 \\
7,8 \\
21,5 \\
14,5 \\
6,1\end{array}$ \\
\hline $\begin{array}{l}\text { Valeur énergétique } \\
\text { - Energie brute } \mathrm{kcal} / \mathrm{kg} \mathrm{MS} \\
\text { - Energie digestible } \mathrm{kcal} / \mathrm{kg} \mathrm{MS} \\
\text { - CUDa Energie }(\%) \\
\text { - Energie métabolisable kcal/kg MS } \\
\text { Valeur azotée } \\
\text { - CUDa Azote }(\%)\end{array}$ & $\begin{array}{c}7066 \pm 266 \\
5403 \pm 135 \\
79,0 \pm 1,75 \\
4792 \pm 119 \\
-\end{array}$ & $\begin{array}{l}6502 \\
5540 \\
83,7 \\
5271 \\
83,7\end{array}$ \\
\hline $\begin{array}{c}\text { Nombre de valeurs } \\
\text { selon les critères }\end{array}$ & $3-9$ & 1 \\
\hline
\end{tabular}

NDF : Neutral Detergent Fiber, ADF : Acid Detergent Fiber, ADL : Acid Detergent Lignin, CUDa : Coefficient d'Utilisation Digestive apparent.

* Synthèse de résultats bibliographiques.

* * Selon Bourdon 1986.

Tableau 5. Composition chimique et valeur nutritive moyennes de tourteaux de colza double 0-BG type pression (moyennes \pm écarts-type de la moyenne).

\begin{tabular}{|c|c|c|}
\hline $\begin{array}{l}\text { Type de colza } \\
\text { Variété }\end{array}$ & \multicolumn{2}{|c|}{$\begin{array}{c}\text { Double 0 } \\
\text { Tandem }\end{array}$} \\
\hline $\begin{array}{c}\text { Type de tourteau } \\
\text { Nombre d'échantillons }\end{array}$ & Double pression & Simple pression \\
\hline $\begin{array}{l}\text { Composition chimique } \\
\text { MS }(\%) \\
\% \text { Matière Sèche } \\
\text { - Cendres brutes } \\
\text { - MAT }(\mathrm{N} \times 6,25) \\
\text { - Matières grasses } \\
\text { - Cellulose brute de Weende } \\
\text { - NDF } \\
\text { - ADF } \\
\text { - ADL }\end{array}$ & $\begin{array}{r}7,1 \\
38,1 \\
8,1 \\
12,8 \\
24,8 \\
17,4 \\
9,9\end{array}$ & $\begin{array}{r}93,5 \pm 0,52 \\
6,4 \pm 0,05 \\
35,9 \pm 0,52 \\
15,3 \pm 0,52 \\
11,1 \pm 0,44 \\
25,7 \pm 0,17 \\
17,4 \pm 0,36 \\
9,6 \pm 0,50\end{array}$ \\
\hline $\begin{array}{l}\text { Valeur énergétique } \\
\text { - Energie brute kcal/kg MS } \\
\text { - Energie digestible kcal/kg MS } \\
\text { - CUDa Energie (\%) } \\
\text { - Energie métabolisable } \mathrm{kcal} / \mathrm{kg} \text { MS }\end{array}$ & $\begin{array}{c}4941 \pm 40 \\
3669 \pm 100 \\
74,3 \pm 2,6 \\
3401 \pm 99\end{array}$ & $\begin{array}{r}5141 \pm 101 \\
4277 \pm 68 \\
83,3 \pm 2,4 \\
4026 \pm 63\end{array}$ \\
\hline $\begin{array}{l}\text { Valeur azotée } \\
\text { - CUDa Azote }(\%) \\
\end{array}$ & $87,5 \pm 1,1$ & $84,3 \pm 1,4$ \\
\hline
\end{tabular}

NDF : Neutral Detergent Fiber, ADF : Acid Detergent Fiber, ADL : Acid Detergent Lignin, CUDa : Coefficient d'Utilisation Digestive apparent.

Toutefois, comme c'est généralement le cas pour les graines oléagineuses, l'huile reste le produit noble le mieux valorisé. C'est pourquoi il peut être envisagé une extraction partielle par pression de l'huile contenue dans les graines sans extraction finale au solvant. L'obtention de tourteaux Expellers, à teneur élevée en huile résiduelle, peut constituer une voie d'utilisation des colzas BG.

\section{3 / Tourteaux de colza double 0-BG obtenus par pression type Expellers}

Les caracteristiques de composition chimique et de valeur nutritive de tourteaux de colza double pression ( $8 \%$ d'huile résiduelle) et simple pression ( $15 \%$ d'huile résiduelle) figurent au tableau 5. Ces tourteaux à teneur élevée en
Tableau 4. Composition chimique et valeur nutritive de la graine entière de colza (moyennes \pm écarts-type de la moyenne). 
Figure 1. Influence du dépelliculage sur la valeur nutritive de tourteaux de colza double $0-B G$ Comparaison aux tourteaux de soja.

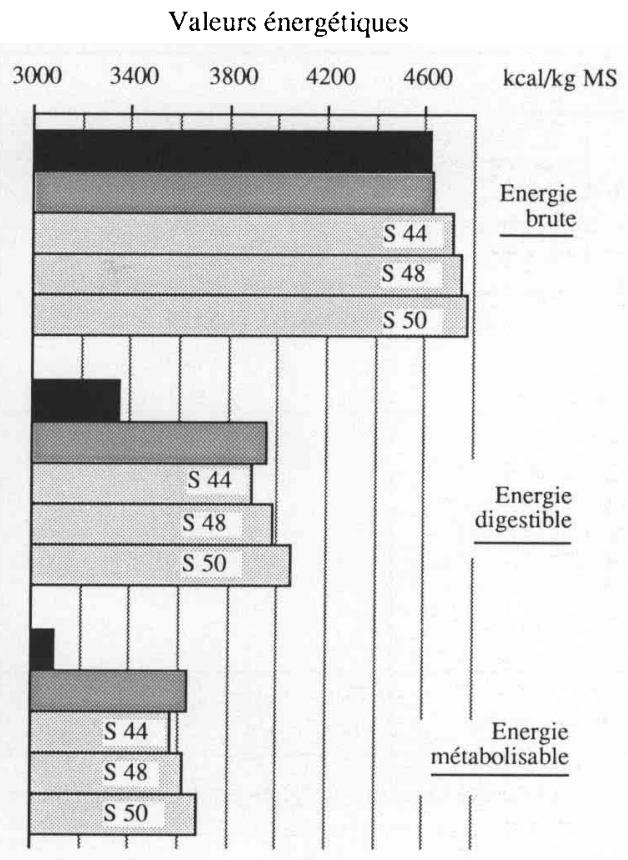

Utilisation digestive

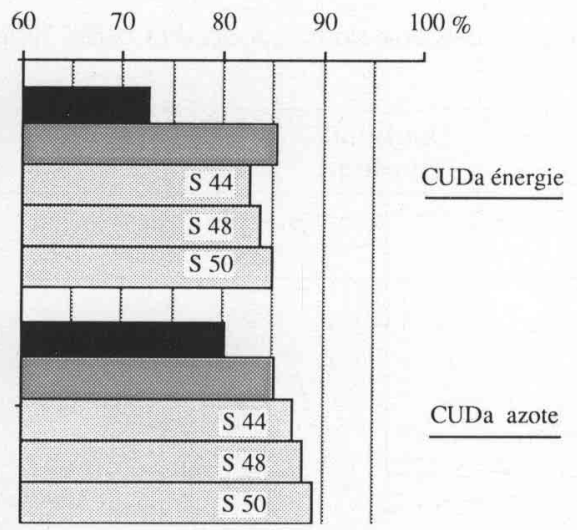

lipides, posent des problèmes de conservation et nécessitent l'addition d'un antioxydant, et un vieillissement. Selon nos résultats expérimentaux, ces deux facteurs associés permettent non seulement de sauvegarder mais également de potentialiser la valeur nutritive des tourteaux de pression. On observe en outre, pour ce type de tourteau, une meilleure digestibilité apparente de la fraction azotée, du fait d'un traitement thermique moins drastique et de la teneur élevée en lipides.

Etant donné le poids économique de la fraction huile, la fabrication de tourteaux de pression ne peut s'envisager que dans des circonstances économiques conjoncturelles spécifiques, l'extraction maximale de l'huile étant généralement pratiquée. C'est pourquoi le « dépelliculage" des graines avant trituration apparaît comme le meilleur moyen d'accroître les valeurs énergétique et azotée du tourteau de colza en réduisant la fraction cellulose indigestible chez le porc

\section{4 / Tourteaux de colza double 0-BG dépelliculés extraits au solvant}

Le dépelliculage améliore la composition chimique et par voie de conséquence la valeur nutritive : en matières azotées : $+15 \%$; en cellulose brute : - $45 \%$; en énergie digestible : + $18 \%$; en énergie métabolisable : $+17 \%$ et respectivement pour l'utilisation digestive apparente de l'énergie et de l'azote : $+18 \%$ et + $6 \%$. Les valeurs nutritives des tourteaux dépelliculés ou non sont présentées sur la figure 1, en comparaison avec celles des tourteaux de soja.

Ainsi pour une composition chimique voisine de celle du tourteau de soja 44 , les tourteaux de colza BG dépelliculés présentent en moyenne pour le porc une valeur nutritive proche de celle du tourteau de soja 48 . Seule la digestibilité de la fraction azotée est plus faible $(85,1$ contre $88 \%)$, associée à une teneur moindre en lysine. En revanche, ils sont mieux pourvus en acides aminés soufrés (méthionine + cystine) et en thréonine (tableau 6).

Tableau 6. Composition moyenne en acides aminés indispensables majeurs des tourteaux de colza. Comparaison à celle d'un tourteau de soja 48 (en $\mathrm{g} / 16 \mathrm{~g} \mathrm{~N}$ ).

\begin{tabular}{|c|c|c|c|c|}
\hline \multirow{3}{*}{ Acides aminés } & \multicolumn{3}{|c|}{ Type de tourteau de colza } & \multirow{3}{*}{$\begin{array}{c}\text { Tourteau } \\
\text { de } \\
\text { soja } 48\end{array}$} \\
\hline & \multicolumn{2}{|c|}{ Non dépelliculé } & \multirow{2}{*}{$\begin{array}{c}\text { Dépelliculé } \\
\text { double } \\
\text { 0-BG }\end{array}$} & \\
\hline & Simple 0-HG & Double 0-BG & & \\
\hline \multirow{3}{*}{$\begin{array}{l}\text { Lysine } \\
\text { Méthionine }\end{array}$} & \multirow{6}{*}{$\begin{array}{l}4,4 \\
1,2\end{array}$} & \multirow{6}{*}{4,7} & \multirow{6}{*}{$2,0\}_{2,6}^{5,3} 3,0$} & \multirow{6}{*}{$\left.\begin{array}{l}1,0 \\
2,0\end{array}\right\}^{6,2} 3,0$} \\
\hline & & & & \\
\hline & & & & \\
\hline Cystine & & & & \\
\hline Thréonine & & & & \\
\hline Tryptophane & & & & \\
\hline $\operatorname{MAT}(\mathrm{N} \times 6,25)$ & 37,9 & 40,7 & 45,0 & 52,0 \\
\hline Nbre de données & 13 & 8 & 6 & - \\
\hline Référence & \multicolumn{3}{|c|}{ Picard et Darcy-Vrillon 1985} & Tables INRA 1984 \\
\hline
\end{tabular}




\section{Conclusion}

Dans la situation actuelle, le tourteau de colza, issu de graines de colza HG, présente des facteurs limitants (inappétence, faible valeur nutritive, toxicité...) qui réduisent au plan pratique à $3,5 \%$ ses possibilités d'incorporation dans les aliments pour le porc à l'engrais. L'utilisation de colza BG associée au dépelliculage des graines permet d'obtenir des tourteaux de meilleure valeur nutritive, dont les taux d'introduction dans les aliments pour le porc sont notablement accrus (jusqu'à 10-20\%). En effet, ces nouveaux tourteaux de colza dépelliculés présentent une valeur nutritive comparable à celle des tourteaux de soja, excepté un léger déficit azoté au plan quantitatif et qualitatif.

\section{Remerciements}

A M. Calet pour son aclion d'incitation et de coordination dans le cadre de la Mission Protéines de 1981 à 1986 .

Nous remercions le Ministère de l'Agriculture, le Ministère de l'Industrie et de la Recherche Scientifique, le CETIOM, l'ONIDOL el SOFIPROTFOL pour les encouragements el l'aide financière à la réalisation de ce programme d'expérimentation.

Egalement nous ne saurions oublier l'ensemble du personnel technique animalier d'expérimentation, de laboratoire ef des ateliers technologiques des différents organismes, auxquels nous adressons nos plus vil's remerciements. Plus particulierement Mme Annie Barbeau et J. Chevallier sont remerciés pour leurs contributions respectives dans la recherche bibliographique et la composition des tableaux.

\section{Références bibliographiques}

AZAIS P., VERMEERSCI G., 1982. Les produits issus du dépelliculage du colza. Rev. Aliment. Anim., 358, 17-21. BAUDET J.J., EVRARD J., 1982. Le tourteau de colza. Les caractéristiques et techmologie. Rev. Aliment. Anim., 352 33-41.

BAUDET J.J., BOURDON D., EVRARD J., LFSSIRE M. 1987. Influence des conditions de cuisson des tourteau de colza sur leur valeur nutritionnelle chez le poulet de chair et le porc à l'engrais. 7th International Rapeseed Congress, Poznan, Pologne, 11-14 mai 1987.

BAUDET J.J., BURGHART P., EVRARD J., 1988. Cahier technique Colza. Tourteaux à basse teneur en glucosinolates (BTG) Ed.CFTIOM, 174, avenue Victor Ilugo, 75116 Paris.

BELL J.M., 1984. Nutrienls and toxicants in rapeseed meal for swine. J. Anikm. Sci., 58, 996-1010

BOURDON D., 1986. Valeur nutritive des nouveaux tour leaux et graines entières de colza à basse teneur on glucosinolates pour le porc à l'engrais. Journées Rech. Porcine en France, 18, 13-23

BOURDON D., BAUDET J.J.. 1983. Valeur nutritive el possibilités d'utilisation du tourteau de colza zérothio dépelliculé pour le porc à l'engrais. 6th Congrès Internalional sur le colza. Paris, 17-19 mai 1983.

BOURDON D., PEREZ J.M., BAUDEI' J.J., 1984. Nutritive value and utilization by the growing-finishing pigs of new types of rapeseed oil meals with low glucosinolate content. In : I1. Sorensen (éd.) World crops : Production. utilization, description, vol. 11. Advances in the production and utilization of cruciferous crops, 177-198. Proceedings of a seminar CEC programme of research on plant protein improvement, Copenhagen, Denmark, 11-13 sept 1984. Martinus Nigholf, Dr W. Junk Publishers, Dorcrechl, The Netherlands.
CALET C.. 1982. Que faut-il penser des constituanls indésirables du tourteau de colzal? Données récentes el conséquences sur les conditions de son utilisation. Rev. Aliment. Anim., 352, 9-18.

CFTIOM, 1988. Oléagineux. Donıées statistiques de production of de consommation (France-CEE-Monde) Mars 1988. 20 p. GETIOM Ed., 174. Avenue Victor Hugo. 75116 Paris.

CHONF F.. 1983. Qualité des produits de transformation du colza double-zéro. CR. Acad. Agr. de France. Séance du 14 décenbre 1983, 1503-1515.

COUDER'T M., 1988. Oléagineux. La nouvelle donne. Rev. Aliment. Anim., 414, 36-41.

EGGUM B.O., OLSEN O., SORENSEN H., 1983. Nutritive value ol rapeseed meal : antinutritional and toxic effects of individual glucosinolates (+ Myrosinases). 6" Congrès International sur le colza, Paris, France, 17-19 mai 1983.

FENWICK G.R.. 1982. The assessment of a new protein source. Rapeseed. Proc. Nutr. Soc., 41, 277-288.

FENWICK G.R., CIJIIS R.F., 1980. Rapeseed meal and its use in poultry diets. A review. Anin. Feed. Sci. Technol. 5. 255-298.

INRA, 1984. Lalimenlation des animaux monogastriques : porc, lapin, volailles. INRA éd., Paris, $228 \mathrm{p}$.

LEE A. Pauline, HILl, R., 1983. Voluntary food intake of growing pigs given diets containing rapeseed meal, from different types and varieties of rape, as the only protein supplement. Br. J. Nutr. 50. 661-671.

LEE A. Pauline, PIT'TMAN S., IIILL R, 1984. The volumtary food intake by growing pigs of diels containing "treated" rapeseed meals or extracts of rapessed meal. Br. J. Nıtr. 52, 159-164.

MENZFI, F., 1983. Untersuchungen über die schildrüsen vergrossernde (strumigene) Wirkung Rapshaltiger Rationen in Abhängigkeil von der Raus sorte dem Glucosinolatgehalt und Füterungs-zusätzen. Inaugural Dissertation zur Erlangung des Doktorgrades der Agrar wissenschaftlichen Fakultät der christian Albrechts, Universität, Kiel. 1983. $143 \mathrm{p}$.

MORICE J., 1983. In nouveau colza : le colza doublezéro. C.R. Acad. $A g r$. de France, Séance du 14 décembre 1983, 1493-1502.

PICARD M., DARCY-VRILLON Béatrice, 1985. Composition en acides aminés du tourtean de colza et digestibilité chez les monogastriques. In Colloque Tourteau de colzí «L'Enjeu ». Paris 12 Juin 1985. 54-73. ONIDOL, éd.. Paris. 277 p.

RUNDGREN Margareta, 1983. Low glucosinolate rapeseed products for pigs. A review. Anim. Feed. Sci. Technol., 9, 239-262.

RUNDGREN Margareta, ASKBRAN'T S., 'l'llOMKE S.. 1985. Nutritional evaluation of low and high-glucosinolate rapeseed meals with pigs, laying hens and rats. Swedish. J. Agri. Res., 15, 61-69.

SAUER W.C., THACKLR P.A., 1986. Apparenl ileal and faecal digestibility of amino acids in barley based diets supplemented with sovabean meal or canola meal for growing pigs. Anim. Feed. Sci. 'Technol, 14, 183-192.

UZZAN $\Lambda$., 1982. Lál lechnologie du colza : de la graine à I'huile et au tourteau. Rev. Aliment. Anim., 352, 29-32.

VERMOREL M., BAUDET J.J., 1987. Valorization of rapeseed meal. 2. Nutritive value of high or low-glucosinolate varieties and effect of clehulling. Reprod. Nulr. Dévelop. 27, 45-55.

VERMOREI, M., FAYET J.C. BAUDE'T J. J, 1978. Valorisation du tourteau de colza. 1. Influence de l'élimination des glucosinolates, du dépelliculage et de l'extraction des alpha galactosides sur sa valeur mutritive. Etude sur le ral en croissance. Anu. Biol. Anim. Bioch. Biophys., $18(6)$ $1393-1412$

VERMOREL, M., HFANEY R.K., FENWICK G.R., 1986. Nutritive value of rapeseed meal : effects of individual glucosinolates. J. Sci. Food. Agric., 37, 1197-1202.

VERMOREL M., DAVICCO Marie-Jeanne, FVRARD J., 1987a. Valorization of rapeseed meal. 3. Effects of glucosinolate content on food intake, weight gain, liver weight and plasma thyrö̈l hormone levels in growing rals. Reprod. Nutr. Dévelop)., 27, 57-66

VERMOREL M., HOCOUEMILLER R., EVRARD J., 1987b. Valorization of rapeseed meal. 5. Effects of sinapine and other phenolic compounds on food intake and nutrient utilization in growing rals. Reprod. Nutr. Jévelop., 27. 781-790. 